\title{
MicroRNA-145 Involves in the Pathogenesis of Renal Vascular Lesions and May Become a Potential Therapeutic Target in Patients with Juvenile Lupus Nephritis
}

\author{
Zhaomin Cai $^{a} \quad$ Wei Xiang $^{c} \quad$ Xiaojie Peng ${ }^{d}$ Yan Ding ${ }^{e}$ Wang Liao ${ }^{f}$ \\ Xiaojie $\mathrm{He}^{\mathrm{b}}$ \\ aDepartment of Clinical Laboratory, People's Hospital of Baoan District of Shenzhen, \\ Shenzhen, PR China; ${ }^{b}$ Laboratory of Pediatric Nephrology, Institute of Pediatrics, Central \\ South University, Changsha, PR China; 'Department of Pediatrics, Hainan Provincial \\ Maternal Hospital, Hainan Province, Haikou, PR China; d Department of Nephrology, Jiangxi \\ Provincial Children's Hospital, Nanchang, PR China; ${ }^{e}$ Department of Dermatology, Hainan \\ Provincial Dermatology Disease Hospital, Haikou, PR China; ${ }^{f}$ Department of Cardiology, \\ Hainan General Hospital, Haikou, PR China
}

\section{Keywords}

Systemic lupus erythematosus - Lupus nephritis - MicroRNA-145 - Renal vascular lesion . Human vascular smooth muscle cells $\cdot$ PDGF-BB

\begin{abstract}
Aims: The current study was conducted with the central objective of investigating the expression of microRNA-145 (miR-145) in renal vascular lesions (RVLs) in juvenile lupus nephritis (JLN) and its possible mechanism. Methods: The clinical data of $49 \mathrm{JLN}$ patients confirmed by renal biopsy were collected and followed by grouping according to the RVLs score after hematoxylin-eosin staining: mild, moderate, and severe groups. In situ hybridization was used to detect the expression of miR-145 in renal vessels which was then being compared among different RVLs groups. Up-LV-miR-145 and LV-miR-NC lentiviral vectors were constructed and transfected into human vascular smooth muscle cells (HVSMCs), respectively. After HVSMCs were treated with $10.0 \mu \mathrm{g} / \mathrm{L}$ platelet-derived growth factor (PDGF)-BB for $24 \mathrm{~h}$, the proliferation, migration, and apoptosis of endothelial cells were detected by MTT, Transwell assay, and flow cytometry, respectively. Western blot was used to detect expression of alpha-smooth muscle actin ( $\alpha$-SM-actin) and osteopontin (OPN). Results: The expression of miR-145 in renal vascular cells was statistically significant. The higher the inner membrane ratio, the lesser the miR-145 expression. After treatment with PDGF-BB, expression of miR-145 in HVSMCs decreased, proliferation and migration ability enhanced, apoptosis decreased, $\alpha$-SM-actin decreased, and OPN increased. The proliferation and migration ability of HVSMCs in the LVmiR-145 group suppressed, apoptosis enhanced, $\alpha$-SM-actin increased, and OPN decreased.
\end{abstract}




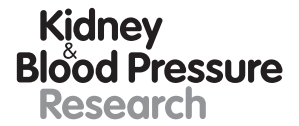

Kidney
Blood Pressure
Research

\begin{tabular}{l|l}
\hline Kidney Blood Press Res 2019;44:643-655 \\
\hline DOI: 10.1159/000500923 & $\begin{array}{l}\text { @ 2019 The Author(s). Published by S. Karger AG, Basel } \\
\text { www.karger.com/kbr }\end{array}$ \\
\hline
\end{tabular}

Cai et al.: miR-145 in RVLs in Patients with JLN

Conclusions: Our study revealed that miR-145 expression decreased with the increase of vascular damage. miR-145 can inhibit proliferation, migration, and differentiation phenotypic transformation of HVSMCs induced by PDGF-BB. miR-145 may be involved in the pathogenesis of RVLs and may be a new target for treatment of RVLs in lupus nephritis.

(C) 2019 The Author(s)

Published by S. Karger AG, Basel

\section{Introduction}

Lupus nephritis (LN) is the most common manifestation and the major cause of death in systemic lupus erythematosus (SLE). LN is featured by glomerular deposition of immune complex accompanied with persistent inflammation [1]. As the main cause of death in patients with lupus, it is reported that LN affects approximately $>70 \%$ of the patients [2]. Statistics have indicated that most patients with nephritis are younger, and males are more susceptible to LN [3]. Moreover, Asian, African Caribbean, and African American ethnicities may suffer more severe nephritis than other ethnic groups [4]. Owing to its complex pathogenesis, LN habitually occurs due to the influence of several factors such as deposition of autoantibodies in glomerulus, production of extracellular matrix proteins, proinflammatory cytokines, and chemokines and activation of complement and macrophages, which may further lead to tubulointerstitial inflammation, tubular damage, and fibrosis [5]. Early diagnosis and treatment of LN aim to prevent the disease from developing into chronic renal failure and renal transplantation [6]. Drug treatment for LN depends on the severity of the disease; therefore, finding reliable LN biomarkers will help to assess disease activity to improve prognosis [7].

MicroRNA (miRNA) plays a role in posttranslational regulation of gene expression through base pairing of complementary sequences and mRNA molecules [8]. In physiological or pathological conditions, such as inflammation and cancer, certain miRNAs can induce mRNA degradation and/or inhibition of protein translation [9]. Accumulating evidence has demonstrated the role of miRNAs in many kinds of kidney diseases including renal cell carcinoma, nephritic syndrome, renal fibrosis, and LN [10]. For example, decreased miR-130b was found in kidney tissues from patients and mouse model of LN, and overexpression of miR-130b could ameliorate IFN $\alpha$-accelerated LN, providing potential therapeutic approaches for LN [11]. MicroRNA-145 (miR-145) was first found in the mouse heart tissue and later reported in human beings, which is located within a $4.09 \mathrm{~kb}$ region on human chromosome 5 (5q32-3) [12]. The miR-143/145 cluster is now considered to be multifunctional miRNAs. For example, as tumor suppressor, both miR-143 and miR-145 can promote cell apoptosis, regulate cell cycle, and inhibit tumor cell growth [13-15]. In addition, miR-145 is recognized as a characteristic tumor suppressor miRNAs with its decreased expression found in many different kinds of cancers, including breast cancer, ovarian cancer, colorectal cancer, and prostate cancer [16-19]. In particular, miR-145 has been reported to act as a proinflammatory molecule in Th2-mediated allergic inflammation [20]. Despite that, the roles of miR-145 in the regulation of immune-related diseases are still largely unknown. In this study, we focused on the roles of miR-145 on renal vascular lesion (RVLs) in children with LN.

\section{Materials and Methods}

\section{Study Subjects}

From January 1, 2012, to January 1, 2017, 49 children with LN hospitalized in the Children's Nephrology Department of Xiangya Second Hospital of Central South University were included in this study, including 11 males and 38 females. The average age was 3-16 years 


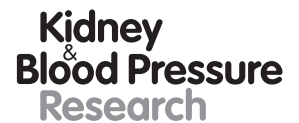

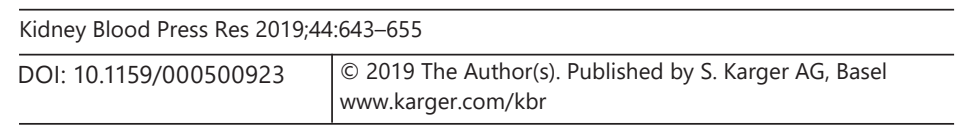

Cai et al.: miR-145 in RVLs in Patients with JLN

(mean age of $8.4 \pm 2.1$ years old). The inclusion criteria were (1) age $\leq 16$ years; (2) in line with the SLE diagnostic criteria recommended by the American rheumatism society in 1997 [21], that is, 4 or more of 11 items were diagnosed as SLE, and renal biopsy was performed to confirm LN; (3) exclude infection, tumor, and connective tissue diseases. Kidney biopsy was performed in all pediatric patients. Kidney biopsy samples were fixed in $10 \%$ formaldehyde buffer, dehydrated with $75 \%$ ethanol gradient, cleaned by xylene, embedded in paraffin, dehydrated, and made into sections with a thickness of $4 \mu \mathrm{m}$. This study was approved by the Ethics Committee of Xiangya Second Hospital of Central South University, and the children's family members signed the informed consent.

\section{Evaluation of RVLS}

Hematoxylin-eosin staining was performed on paraffin sections of renal biopsy tissues. The characteristics of vascular lesions were observed by selecting the median or proximal sagittal section of the small/fine renal artery under optical microscope with image photographed ( $\times 400$ ). Image-Pro Plus 6.0 (Media Cybernetics, Inc., Rockville, MD, USA) image analysis system was used for measuring the area of membrane and the total area of blood vessels. The ratio of inner membrane area to vascular area presented the thickening degree of inner membrane (the ratio of inner membrane area). The mild group showed the ratio of inner membrane $\leq 0.6$, the moderate group was between 0.63 and 0.75 , and the severe group was $\geq 0.75$.

\section{In situ Hybridization}

In situ hybridization was used to detect the miR-145 expression in renal vessels. The probe sequence is CTGGGAAAACTGGACCGTGAGG (purchased from Changsha AI Jia Biotechnology Co., Ltd.). Paraffin section of renal biopsy tissue was dewaxed, placed in $3 \% \mathrm{H}_{2} \mathrm{O}_{2}$ at room temperature for $10 \mathrm{~min}$, digested by pepsin diluted with $3 \%$ citric acid freshly at $37^{\circ} \mathrm{C}$ for $30 \mathrm{~min}$, washed with $0.5 \mathrm{~mol}$ PBS for 3 times, 5 min each time, and then washed by distilled water 1 time for $5 \mathrm{~min}$. After prehybridization at $37^{\circ} \mathrm{C}$ for $2 \mathrm{~h}$, the section was added with a mixture of diluted hybridization solution and probe $(1: 500)$ for hybridization at $55^{\circ} \mathrm{C}$ for overnight. The sections were then washed by $2 \times$ SSC for 3 times, blocked for $30 \mathrm{~min}$ at $37^{\circ} \mathrm{C}$, and blocked for another $2 \mathrm{~h}$ at room temperature (all reagents were purchased from Wuhan Boster Biological Technology Co., LTD., Hubei, China). Subsequently, the section was developed with DAB (purchased from Fuzhou Maixin Biotechnology Development Co., Ltd., Fuzhou, China), redyed by hematoxylin, dehydrated, cleaned, and mounted by neutral gum. The expression characteristics of miR-145 were observed under optical microscope under 200 times and photographed. Image-Pro Plus 6.0 (Media Cybernetics, Inc., Rockville, MD, USA) image analysis system was used to measure the average gray scale of the positive area and the positive area of the miR-145. The relative expression of miR-145 was (miR-145 positive area/[mean gray scale of positive area of miR-145 $\times$ inner membrane area]) $\times 1,000$.

\section{Human Vascular Smooth Muscle Cells Culture}

Human vascular smooth muscle cells (HVSMCs) (purchased from the Institute of Cell Research, Shanghai Academy of Sciences, Shanghai, China) were cultured at $37^{\circ} \mathrm{C}$ with $5 \%$ $\mathrm{CO}_{2}$ with DMEM $(1 \times)$ high glucose medium supplemented with $10 \%$ fetal bovine serum, $100 \mathrm{U} / \mathrm{mL}$ penicillin, 4,500 mg/L glucose, $10 \mathrm{U} / \mathrm{mL}$ streptomycin, and sterile mycoplasmafree (purchased from Genom biopharmaceutical technology co., Ltd., Hangzhou, China). Cell passage was conducted when cell confluency reached $80-90 \%$.

\section{Lentivirus Transfection and Grouping}

HVSMCs were divided into LV-miR-145 group, empty vector control group (LV-NC group), and blank control group (blank group). HVSMCs in logarithmic phase were digested by $0.25 \%$ 


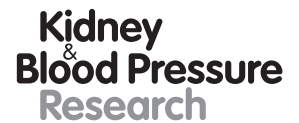

\begin{tabular}{l|l}
\hline Kidney Blood Press Res 2019;44:643-655 \\
\hline DOI: 10.1159/000500923 & $\begin{array}{l}\text { @ 2019 The Author(s). Published by S. Karger AG, Basel } \\
\text { www.karger.com/kbr }\end{array}$ \\
\hline
\end{tabular}

Cai et al.: miR-145 in RVLs in Patients with JLN

EDTA-containing trypsin and centrifuged for $10 \mathrm{~min}$ at $1,000 \mathrm{~g}$. After counting under microscope, the cells were inoculated in the 6-well plate and incubated for $24 \mathrm{~h}$. After incubation, the cells adhered to the wall and returned to normal growth state. Sterile straw was used to aspirate culture medium, and the cells were washed by PBS, followed by the addition of $1 \mathrm{~mL}$ lentiviral transfection reagent (negative control virus or miR-145 overexpression virus). The blank control group did not add. After $12 \mathrm{~h}$ incubation, the transfection reagent was replaced by normal medium and the incubation was continued. After $72 \mathrm{~h}$, the transfected cells were observed under fluorescence microscope. If green fluorescence could be seen, the transfected cells could be screened by purinomycin for 2-3 weeks until the transfected cells could grow steadily. HVSMCs from the LV-miR-145 group, the LV-NC group, and the blank group were cultured in serum-free medium for $24 \mathrm{~h}$ after $10.0 \mu \mathrm{g} / \mathrm{L}$ platelet-derived growth factor (PDGF)-BB treatment. Lentiviral vectors Up-LV-miR-145 (LV-miR-145 group) and LV-miR-NC (LV-NC group) were constructed by Shanghai GeneChem Co., Ltd. (Shanghai, China).

\section{Fluorescent Quantitative Reverse Transcription-Polymerase Chain Reaction}

The cells were incubated in the12-well plate, and each group had 3 duplicated wells. When the cell confluency reached 80\%, $1 \mathrm{~mL}$ Trizol (Invitrogen, Carlsbad, CA, USA) was added into each well to lyse the cells for $15 \mathrm{~min}$ and $200 \mathrm{~mL}$ chloroform was added to extract the total RNA. The concentration and purity of RNA were determined by enzyme labeling (Eppendorf, Hamburg, Germany). After 500 ng of RNA was taken from each well sample, the cDNA was synthesized by reverse transcription. Samples were added according to the polymerase chain reaction (PCR) preparation system and fluorescence quantitative reverse transcription-PCR was performed. The PCR reaction conditions are as follows: the template degeneration was maintained at $15 \mathrm{~min}$ at $95^{\circ} \mathrm{C}$; PCR cycle template denatured for $20 \mathrm{~s}$ at $94^{\circ} \mathrm{C}$; annealing at $60^{\circ} \mathrm{C}$ and extended for $34 \mathrm{~s}$, with a total of 40 cycles. The melting curve is at $95^{\circ} \mathrm{C}$ for $15 \mathrm{~min}$, at $60^{\circ} \mathrm{C}$ for $1 \mathrm{~min}$, and $95^{\circ} \mathrm{C}$ for $15 \mathrm{~min}$. The $2^{-\Delta \Delta \mathrm{C}}$ method was used for relative quantification. The upstream primer of miR-145 was 5'-CAGTGCGTGTCGTGGAGT-3', the downstream primer was 5'-AGGTCCAGTTCCCAGG-3', and U6 was used as internal reference with upstream primer of 5'-CTCGCTTCGCA-3', and downstream primer of ACGCTTCACGAATGGT.

\section{3-(4,5-Dimethyl-2-Thiazolyl)-2,5-Diphenyl-2-H-Tetrazolium Bromide (MTT) Colorimetric Experiment}

When the cell confluency reached $80 \%$, the cells were washed by PBS for 2 times, digested by regular trypsin, and titrated to form a single cell suspension. Cells were counted with cell counter. The cells were then inoculated into the 96 -well plate with a density of $3 \times 10^{3}$ to $6 \times$ $10^{3}$ cells/well (100 $\mu \mathrm{L}$ per well) for culture for $12-24 \mathrm{~h}$. Subsequently, cells were added with different concentrations of PDGF-BB (0, 5.0,10.0, $20.0 \mu \mathrm{g} / \mathrm{L}$; final volume of $200 \mu \mathrm{L}$; purchased from Shanghai First Biochemical Pharmaceutical Co., Ltd., Shanghai, China) for incubation in $5 \% \mathrm{CO}_{2}$ incubator at $37^{\circ} \mathrm{C}$. Six duplicated wells were set. After that, the cells were added with $20 \mu \mathrm{L}$ MTT solution ( $5 \mathrm{mg} / \mathrm{mL}$, Sigma-Aldrich Chemical Company, St Louis, MO, USA) to develop, followed by further incubation in a $5 \% \mathrm{CO}_{2}$ incubator at $37{ }^{\circ} \mathrm{C}$ for $4 \mathrm{~h}$. The culture was terminated and the culture medium was discarded. DMSO was added to each well at a dose of $150 \mu \mathrm{L}$, shaking gently for $10 \mathrm{~min}$ to promote crystallization dissolution. The absorbance values at $570 \mathrm{~nm}$ of each well were determined by enzyme-labeled instrument (BioRad, USA) after a time period of 24,48 , and $72 \mathrm{~h}$. The experiment was repeated 3 times.

\section{Transwell Assay}

HVSMCs at absolute growth stage in the LV-miR-145 group, the LV-NC group, and the blank group as well as the control group (cells without treated by $10.0 \mu \mathrm{g} / \mathrm{L}$ PDGF-BB) were treated with $0.25 \%$ trypsin. The cell concentration was adjusted to $2.5 \times 10^{4} / 100 \mu \mathrm{L}$ and the 


\section{Kidney \\ Blood Pressure \\ Research}

\begin{tabular}{l|l}
\hline Kidney Blood Press Res 2019;44:643-655 \\
\hline DOI: 10.1159/000500923 & $\begin{array}{l}\text { @ 2019 The Author(s). Published by S. Karger AG, Basel } \\
\text { www.karger.com/kbr }\end{array}$ \\
\hline
\end{tabular}

Cai et al.: miR-145 in RVLs in Patients with JLN
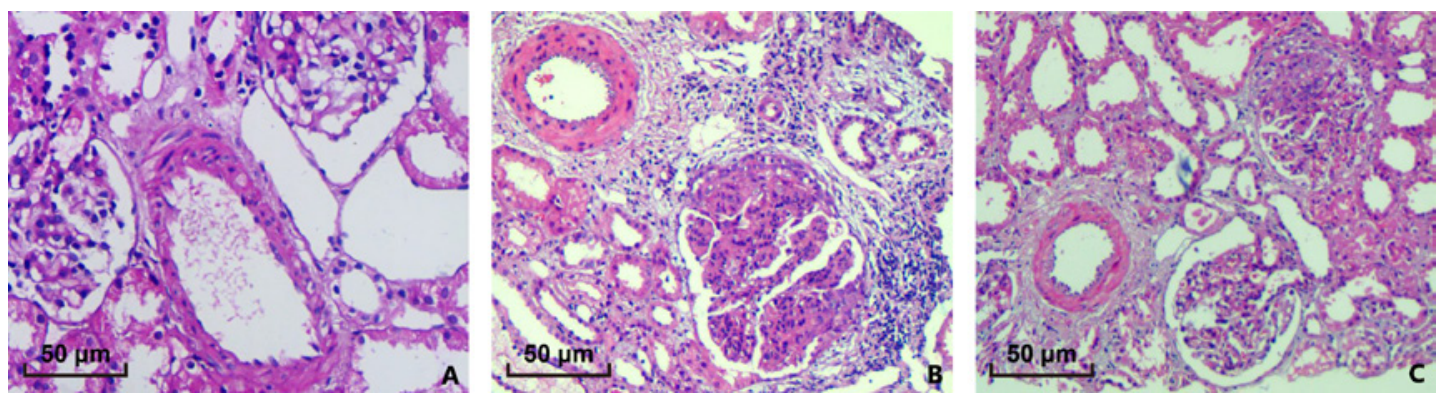

Fig. 1. Damage of glomerular and vascular detected by hematoxylin-eosin staining $(\times 400)$. A Mild group. B Moderate group. C Severe group. Vascular damage manifested as evident intimal exfoliation and thickening of the inner membrane. With the increase of thickening of inner membrane, the degree of intimal exfoliation aggravated and the loss of vascular integrity aggravated.

cells were inoculated into the apical chamber of Transwell chamber (Corning Company), and then $500 \mu \mathrm{L}$ DMEM (Beijing solarbio science and technology Co., Ltd., Beijing, China) containing $10 \%$ fetal bovine serum was added into the basolateral chamber for incubation in $5 \% \mathrm{CO}_{2}$ incubator at $37^{\circ} \mathrm{C}$ for $24 \mathrm{~h}$. After that, the migration chamber was removed, the medium was discarded, and the cells that did not cross the membrane inside the chamber were gently wiped off with a cotton swab, followed by PBS washing for 3 times. The cells were then fixed with $4 \%$ paraformaldehyde for 20 min, washed by PBS for 3 times, and stained by $0.1 \%$ crystal violet for $15 \mathrm{~min}$. Under optical microscope, 5 (200x) visual fields were randomly selected to count the number of cells passing through the membrane and photographed.

\section{Flow Cytometry}

HVSMCs from the LV-miR-145 group, the LV-NC group, and the blank group as well as the control group were inoculated into the 6-well plate and detected according to the instructions of apoptosis detection kit (Nanjing KeyGen Biotech Co., Ltd., Nanjing, China). Cells were digested with $0.25 \%$ trypsin (without EDTA), centrifuged, collected, washed with PBS 3 times, then added with $500 \mu \mathrm{L}$ precooled $1 \times$ binding buffer and with a mixture of $5 \mu \mathrm{L}$ Annexin-VFITC and 2.5 $\mu \mathrm{L}$ PI. The cells were detected by flow cytometry (BD FACSArial I cell sorter, BD Biosciences). Results showed that the left lower quadrant (Q4) was representative of the healthy living cells (FITC-/PI-); the right lower quadrant (Q3) represented the early apoptotic cell, showing (FITC+/PI-); the upper right quadrant (Q2) indicated the late stage of necrosis and apoptosis (FITC+/PI+); and the apoptosis rate represented by the percentage of early apoptosis (Q3) + late apoptosis (Q2).

\section{Western Blot Analysis}

Protein was extracted from the cell samples. The protein samples were added with $5 \times$ loading buffer and boiled in water for $10 \mathrm{~min}$. SDS-PAGE protein electrophoresis was then carried out. After electrophoresis, the protein was transferred onto PVDF membrane by wet transfer method. The membrane was then sealed in 5\% evaporated milk-TBST at room temperature for $2 \mathrm{~h}$. After sealing, the PVDF membrane was incubated with the rabbit anti-human alpha-smooth muscle actin ( $\alpha$-SM-actin; osteopontin [OPN], 1:1,000, Cell Signaling, Boston, MA, USA) at $4{ }^{\circ} \mathrm{C}$ overnight. After that, the membrane was washed with TBST for 3 times (each for $10 \mathrm{~min})$ and added with horseradish peroxidase labeled goat anti-rabbit IgG (1:5,000, Beijing Kangwei Biotech Co., Ltd., Beijing, China) for incubation at room temperature for $1 \mathrm{~h}$. The membrane was developed via chemiluminescence, fixed, and analyzed with $\beta$-actin as reference. 


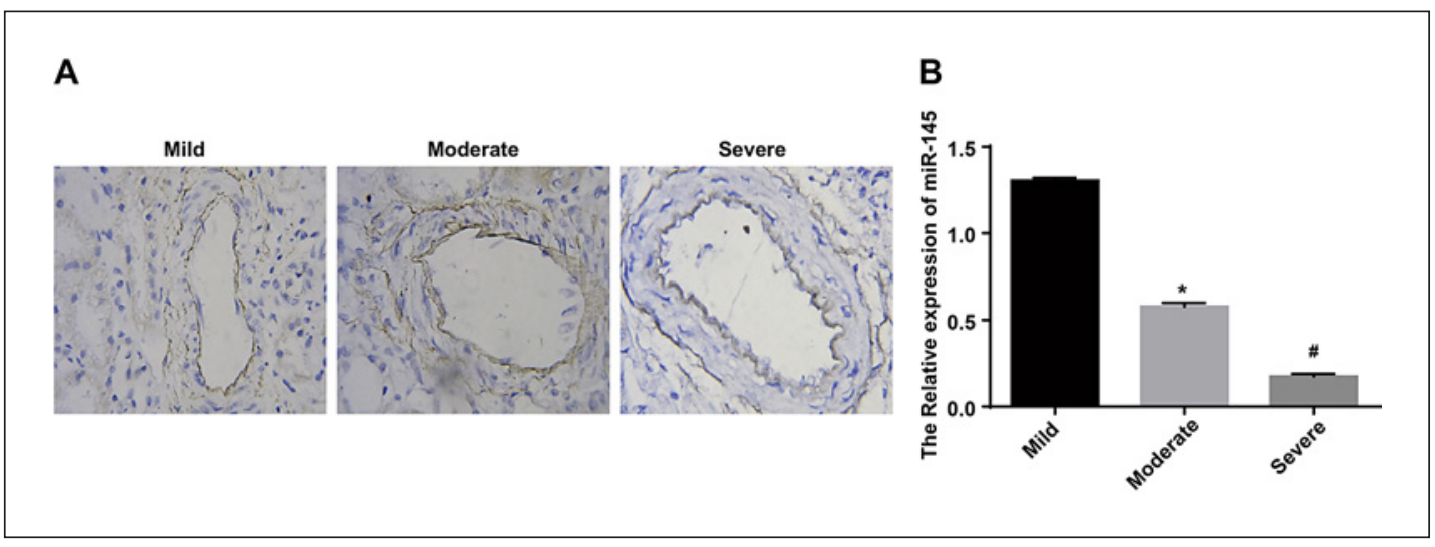

Fig. 2. Expression of miR-145 in renal vessels with different diameters $(\times 400)$. A The mild group with basically normal inner membrane; the moderate group with different thickness of inner membrane; the severe group with obviously thickened inner membrane. B Expression of miR-145 in different ratios of inner membrane; * $p<0.05$, compared with the mild group; ${ }^{*} p<0.05$, compared with the moderate group. miR-145, microRNA-145.

Fig. 3. Effect of different concentrations of PDGF-BB on proliferation of HVSMCs by MTT assay.

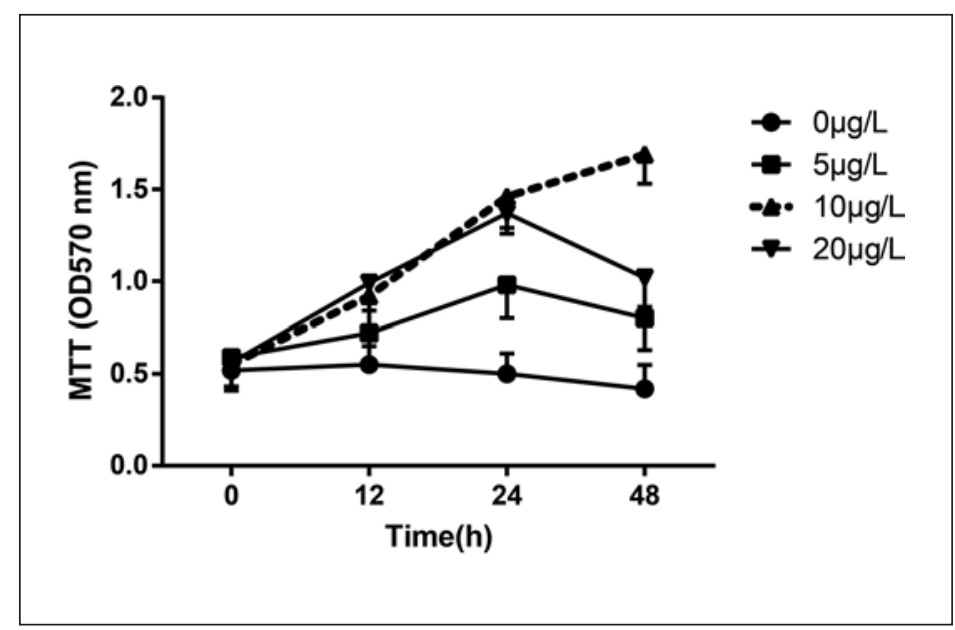

\section{Statistical Analysis}

SPSS 19 software was used for data analysis. The measurement data were presented as mean \pm SD. One-way analysis of variance was used for comparison among multiple groups (the homogeneity of variance was tested before analysis), and LSD $t$ test was used for pairwise comparison of multiple groups/means. Value of $p<0.05$ showed significant difference.

\section{Results}

Observation of RVLs in Fourty-Nine Children with LN

RVLs in children with LN mainly presented as the abscission of intima and thickening of the inner membrane. In the mild group ( $n=4$ cases), the endovascular intima was intact and endothelial cell hyperplasia was obvious. In the moderate group ( $n=17$ cases), some of the intima were damaged, but some of the intima were asymmetrical hyperplasia, and the thickness of the inner membrane was different. In the severe group ( $n=28$ cases), the intima 
Fig. 4. Real-time PCR was used to detect the expression of miR145 in HVSMC cells treated with $10 \mu \mathrm{g} / \mathrm{L}$ PDGF-BB for $24 \mathrm{~h}$. * Indicates that compared with the control group, $p<0.05$. miR-145, microRNA-145.

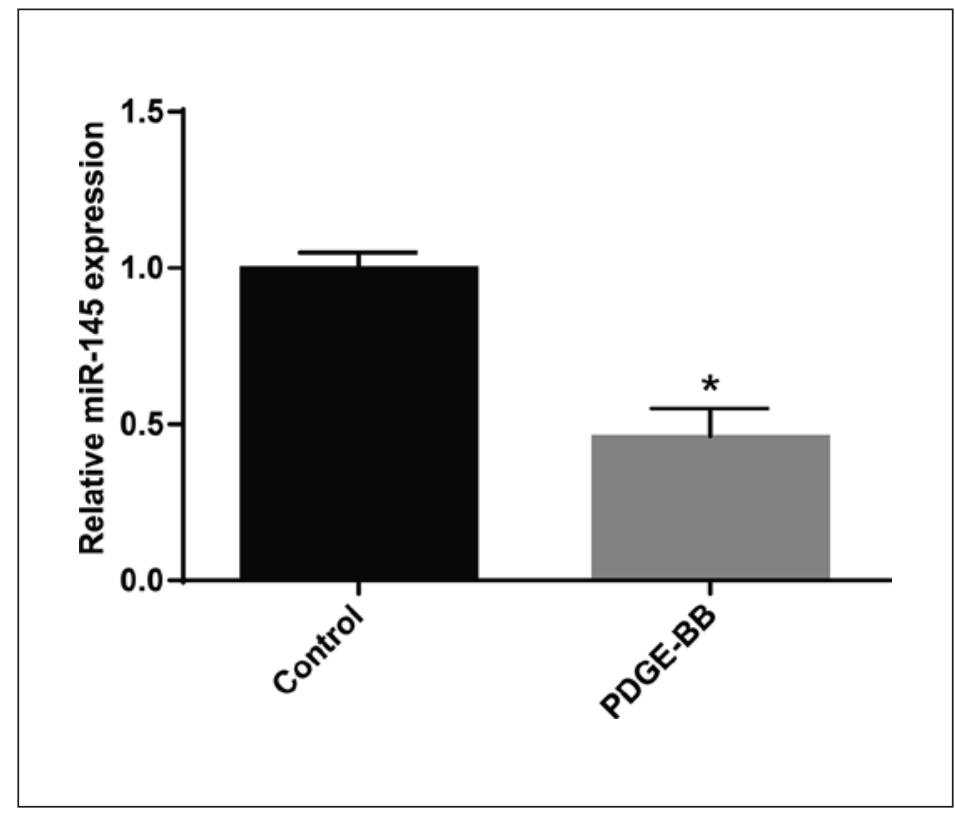

was destroyed severely, and the vascular endothelial cells were found to be exfoliated and severe stenosis was observed in the lumen of the vessel; the inner membrane was obvious hyperplasia, and most of the smooth muscle cells were spindle shaped. It can be seen that the higher the degree of thickening of the inner membrane, the greater the degree of endometrial exfoliation and vascular integrity loss (Fig. 1).

\section{miR-145 Is Related to the Degree of RVLs in LN Children}

The miR-145 expression was then detected to explore the relationship between miR-145 and degree of RVLs. miR-145 was mainly expressed in epithelial cells of proximal convoluted tubules and vascular smooth muscle cells of renal vessels (Fig. 2A). In situ hybridization experiment showed that the higher the ratio of inner membrane, the lesser the expression of miR-145 was (Fig. 2B). It is indicated that the miR-145 expression was related to the degree of RVLs in LN children.

\section{Effect of PDGF-BB on Hyperplasia of HVSMCS}

In order to study the mechanism of renal vascular dysplasia in LN children, we used 0 , 5.0, 10.0, $20.0 \mu \mathrm{g} / \mathrm{L}$ PDGF-BB to treat HVSMCs. MTT assay was used to detect the proliferation of HVSMCs. The results showed that the proliferation of VSMCs increased gradually with the increase of PDGF-BB concentration at 12 and $24 \mathrm{~h}$. But at $48 \mathrm{~h}$, the OD value of $10.0 \mu \mathrm{g} / \mathrm{L}$ PDGF-BB group increased continuously, while that of 5.0 and $20.0 \mu \mathrm{g} / \mathrm{L}$ PDGF-BB groups decreased (Fig. 3). In the following experiment, we chose $10.0 \mu \mathrm{g} / \mathrm{L}$ PDGF-BB to intervene VSMCs for $24 \mathrm{~h}$ as the best treatment condition.

\section{miR-145 Expression in HVSMCs after PDGF-BB Treatment}

After HVSMC was treated with $10.0 \mu \mathrm{g} / \mathrm{L}$ PDGF-BB for $24 \mathrm{~h}$, the miR-145 expression was detected by real-time PCR. The results showed that the miR-145 expression in the PDGF-BB stimulation group was significantly lower than that in the control group (without PDGF-BB; Fig. $4, p<0.05$ ). It is indicated that the miR-145 expression was negatively regulated by PDGFBB. 
A
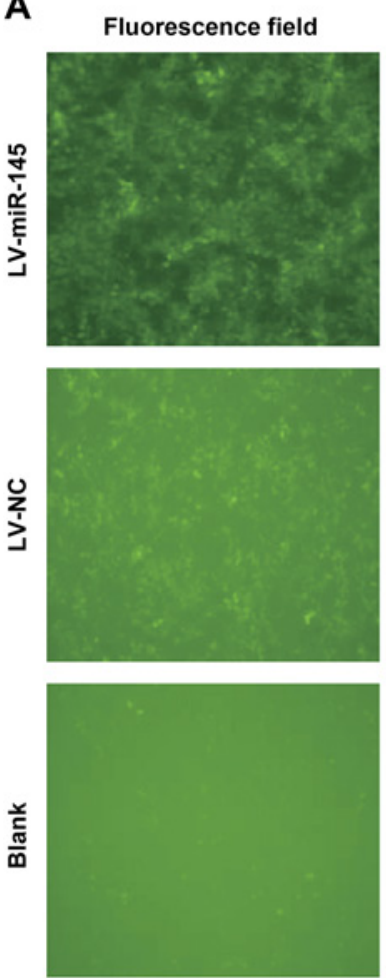

Light field
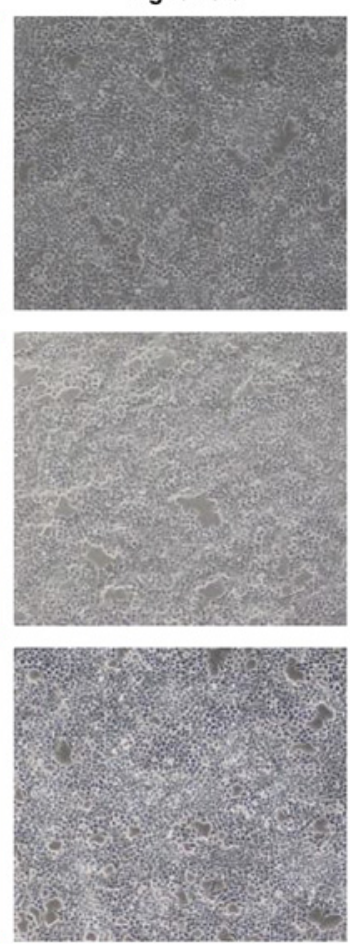

B

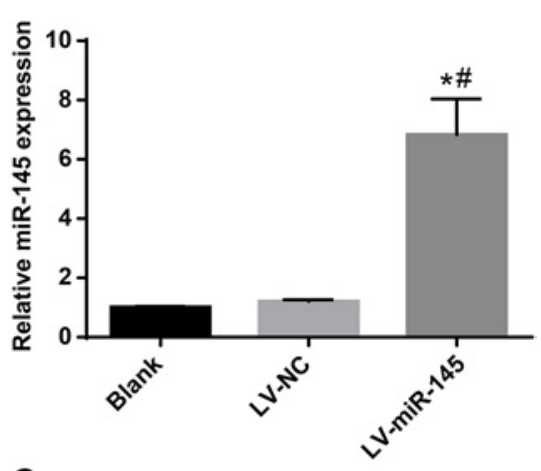

C

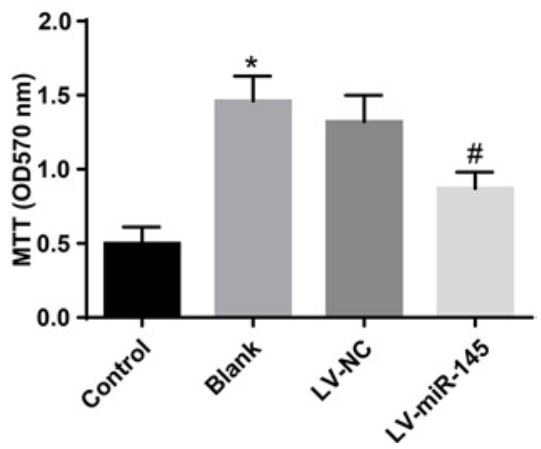

Fig. 5. Validation of lentivirus transfection efficiency and effect of miR-145 on HVSMC proliferation induced by PDGF-BB. A The efficiency of virus infection in HVSMCs was observed $(\times 120)$. B Reverse transcriptionPCR was used to detect the expression of miR-145 in the LV-miR-145, blank and LV-NC groups $72 \mathrm{~h}$ after lentivirus transfection. C After stable transfection of HVSMCs with $10.0 \mu \mathrm{g} / \mathrm{L}$ PDGF-BB for $24 \mathrm{~h}$, the proliferation of HVSMCs in the LV-miR-145, blank, LV-NC, and control groups was detected by MTT assay. * Compared with the control group, $p<0.05$; " compared with the LV-NC or blank groups, $p<0.05$. miR-145, microRNA-145.

\section{Upregulation of miR-145 Inhibits Proliferation of HVSMCs Induced by PDGF-BB}

After HVSMCs were transfected by lentivirus for $72 \mathrm{~h}$, obvious green fluorescence could be seen under fluorescent microscope (Fig. 5A). The miR-145 expression in the LV-miR-145 group was 6.7 times higher than that in the control group $(p<0.05), 5.6$ times higher than that in the LV-NC group ( $p<0.05)$, but there was no significant difference in the miR-145 expression between the blank and LV-NC groups ( $p>0.05$; Fig. 5B).

In order to study the effect of miR-145 on the proliferation of HVSMCs induced by PDGF-BB, HVSMCs were stably transfected with $10.0 \mu \mathrm{g} / \mathrm{L}$ PDGF-BB for $24 \mathrm{~h}$. The survival rates of cells in the LV-miR-145, LV-NC, blank, and control groups were measured by MTT assay. The results showed that the proliferation of HVSMCs was decreased in the LV-miR-145 group compared with the blank and LV-NC groups $(p<0.05)$. However, there was no significant difference in proliferation of HVSMCs between the blank group and the LV-NC group (Fig. 5C). The proliferation of HVSMCs induced by PDGF-BB was inhibited by transfection of miR-145. 


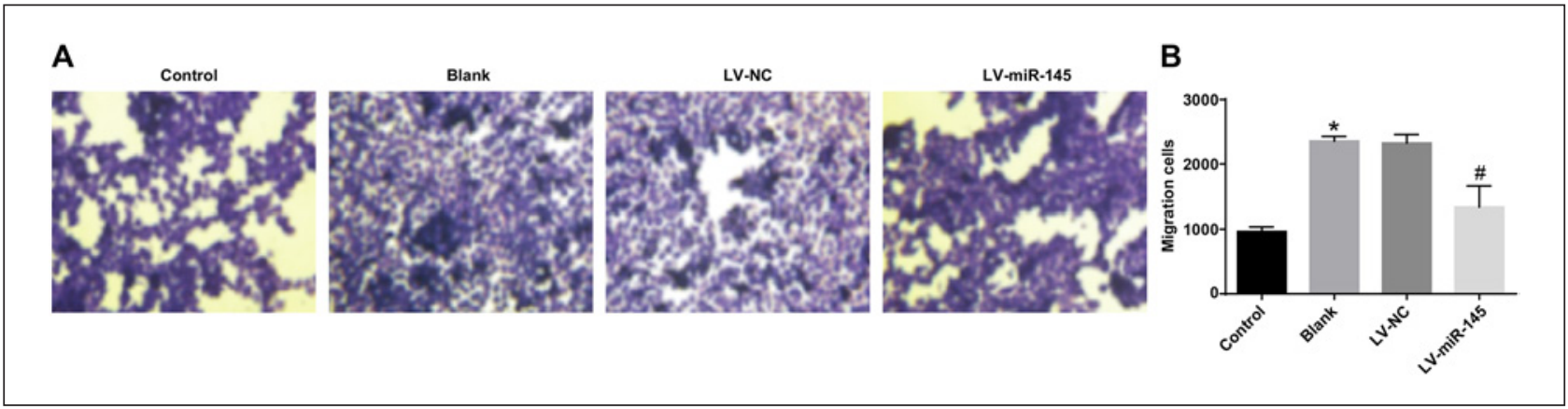

Fig. 6. Effect of miR-145 on migration ability of HVSMCs induced by PDGF-BB. After stable transfection of HVSMCs with $10.0 \mathrm{\mu g} / \mathrm{L}$ PDGF-BB for $24 \mathrm{~h}$, the migration ability of HVSMCs in the LV-miR-145, blank, LV-NC, and control groups was detected by Transwell assay. * Compared with the control group, $p<0.05$; ${ }^{*}$ com- $^{-}$ pared with the LV-NC or blank groups, $p<0.05$. miR-145, microRNA-145.
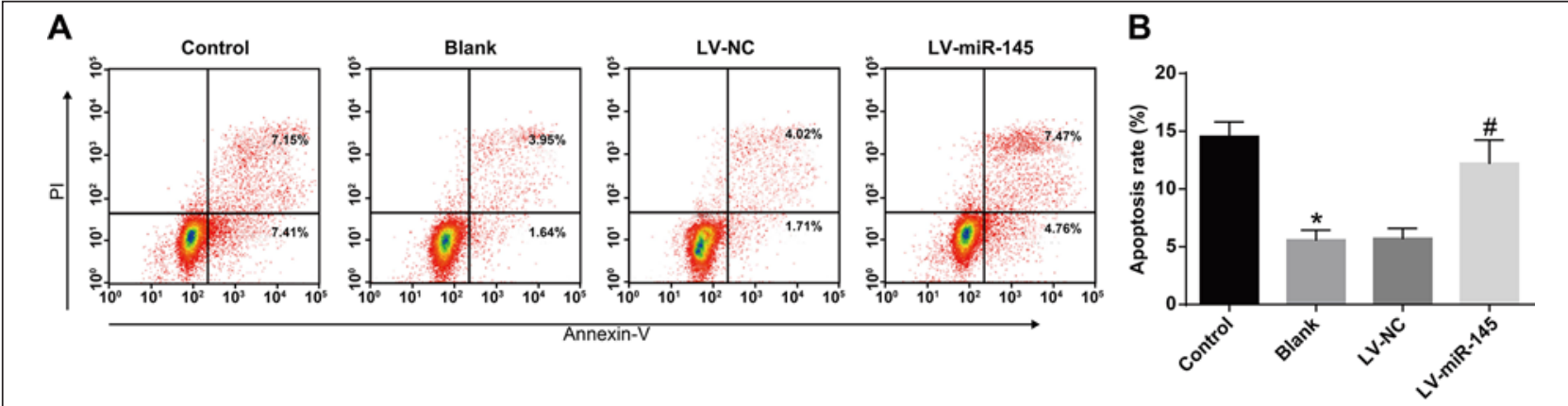

Fig. 7. Effect of miR-145 on apoptosis in HVSMCs induced by PDGF-BB. After stable transfection of HVSMCs, $10.0 \mu \mathrm{g} / \mathrm{L}$ PDGF-BB was used for treating the cells for $24 \mathrm{~h}$. The apoptosis of HVSMCs in the LV-miR-145, blank, LV-NC, and control groups was detected by flow cytometry. * Compared with the control group, $p<$ $0.05 ;{ }^{*}$ compared with the LV-NC or blank groups, $p<0.05$. miR-145, microRNA-145.

Fig. 8. Effect of miR-145 on differentiation phenotype transformation of HVSMCs induced by PDGFBB. After stable transfection of HVSMCs with $10.0 \mu \mathrm{g} / \mathrm{L}$ PDGF-BB for $24 \mathrm{~h}$, the changes of $\alpha$-SMactin and OPN in the LV-miR-145, blank, LV-NC, and control groups were detected by Western blot analysis. * Compared with the control group, $p<0.05$; ${ }^{\#}$ compared with the LV-NC or blank groups, $p<0.05$. $\alpha$-SM-actin, smooth muscle actin; OPN, osteopontin; miR-145, microRNA-145.

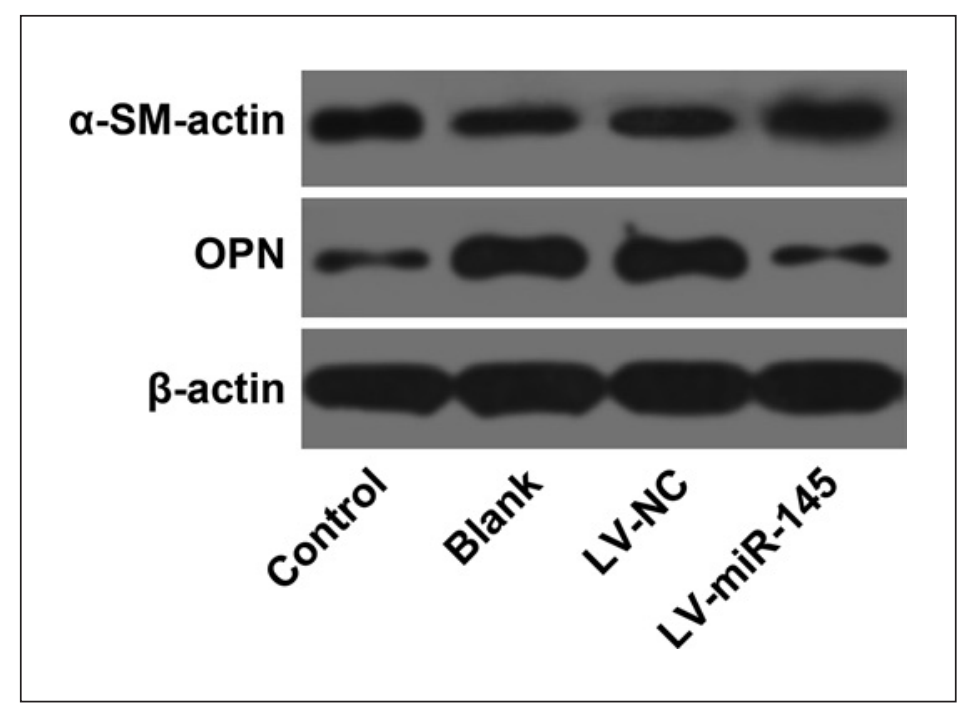




\section{Kidney \\ Blood Pressure \\ Research}

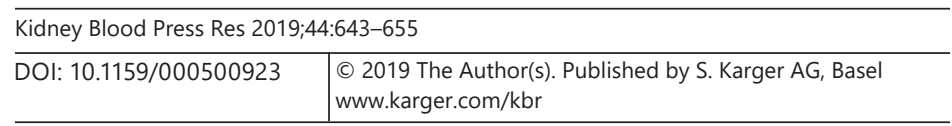

Cai et al.: miR-145 in RVLs in Patients with JLN

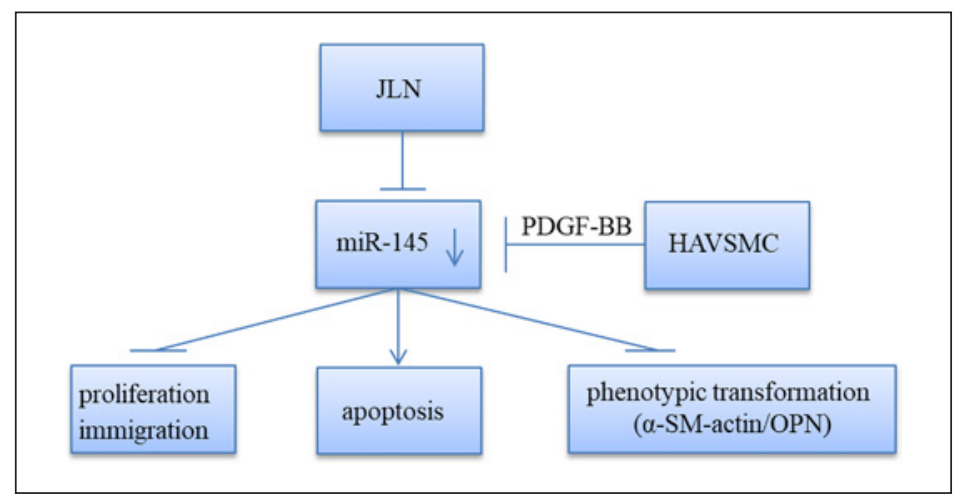

Fig. 9. Possible molecular mechanisms of miR-145 as a potential therapeutic target in patients with JLN. JLN, juvenile lupus nephritis; HVSMCs, human vascular smooth muscle cells; miR145, microRNA-145; $\alpha$-SM-actin, smooth muscle actin; OPN, osteopontin.

Upregulation of miR-145 Inhibits Migration of HVSMCs Induced by PDGF-BB

The migrative ability of HVSMCs in the LV-miR-145, LV-NC, blank, and control groups was detected by Transwell assay. The migrative ability of HVSMCs in the blank group was significantly enhanced than that in the control group $(p<0.05)$. Compared with the blank and LV-NC groups, the migrative ability of HVSMCs decreased in the LV-miR-145 group $(p<0.05)$, while there was no significant difference in migrative ability of HVSMCs between the blank and LV-NC groups ( $p>0.05$; Fig. 6). The results showed that migration of HVSMCs induced by PDGF-BB was inhibited by transfection of miR-145.

Upregulation of miR-145 Promotes Apoptosis in HVSMCs Induced by PDGF-BB

After stable transfection, HVSMCs were treated with $10.0 \mu \mathrm{g} / \mathrm{L}$ PDGF-BB for $24 \mathrm{~h}$, and the apoptosis was then detected by flow cytometry. The results showed that the apoptosis rate of HVSMCs in the blank group was significantly lower than that in the control group $(p<0.05)$. Compared with the blank and LV-NC groups, the apoptosis rate of HVSMCs in the LV-miR-145 group increased significantly $(p<0.05)$. However, there was no significant difference in apoptosis rate of HVSMCs between the control group and the LV-NC group (Fig. 7). These findings revealed that transfection of miR-145 into HVSMCs induced by PDGF-BB could promote apoptosis of HVSMCs.

Upregulation of miR-145 Inhibits Differentiation Phenotype Transformation of HVSMCs Induced by PDGF-BB

After stable transfection, $10.0 \mu \mathrm{g} / \mathrm{L}$ PDGF-BB was used for treating HVSMCs for $24 \mathrm{~h}$. The expression of $\alpha$-SM-actin and OPN was measured by Western blot analysis. The results showed that the expression of $\alpha$-SM-actin was lower, while OPN was higher in the blank group than those in the control group. Compared with the blank group and the LV-NC group, $\alpha$-SMactin increased and OPN decreased in the LV-miR-145 group $(p<0.05)$. There was no significant difference in expression of $\alpha$-SM-actin and OPN between the blank and LV-NC groups (Fig. 8). The above results demonstrated that miR-145 could prevent differentiation phenotype transformation of HVSMCs induced by PDGF-BB to a certain extent.

\section{Discussion}

LN is characterized by autoantibody- induced renal damage in patients with SLE and acted as a crucial factor in endothelial dysfunction and accelerated atherosclerosis presented by patients with SLE $[22,23]$. In the recent years, miRNAs have been found to play a role in 


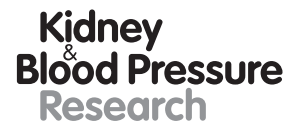

Kidney
Blood Pressure

Research \begin{tabular}{l|l}
\hline Kidney Blood Press Res 2019;44:643-655 \\
\hline DOI: 10.1159/000500923 & $\begin{array}{l}\text { @ } 2019 \text { The Author(s). Published by S. Karger AG, Basel } \\
\text { www.karger.com/kbr }\end{array}$ \\
\hline
\end{tabular}

Cai et al.: miR-145 in RVLs in Patients with JLN

many kinds of cellular processes, including differentiation and development of autoimmune diseases [24]. Thus, in our study, we enrolled 49 pediatric patients with LN for investigating the regulatory role of miR-145 on RVLs in LN.

First, RVLs conditions were observed, and pediatric patients with LN mainly presented with the abscission of intima and thickening of the inner membrane. Evidence has been presented suggesting that RVLs including vascular endothelial injury, noninflammatory necrosis, and cellulose degeneration, which are known as the commonest causes of renal dysfunction, poor treatment, and prognosis [25]. Previous study also clarified that the renal vascular damage observed in LN patients can be caused by intima hyperplasia, or unevenly thickening of tunicae media vasorum due to intima damages [21,26]. In addition, the presence of renal vascular changes directly affects the prognosis of patients with LN [27]. Then, the expression of miR-145 in intrarenal vessels was evaluated, and the results in our present study revealed that the higher the ratio of inner membrane, the lesser the expression of miR-145 was, and the expression of miR-145 was related to the degree of RVLs in LN children. Active SLE in the patients can activate the immune system, leading to disruption of epithelial cells, which directly affect clinical development, treatment, and prognosis of patients with LN $[28,29]$. In recent years, new clues about the development and self-sustainability of LN have emerged, pointing directly to the multistep inflammatory process triggered by antichromatin antibodies (most notably anti-DNA and antinucleosome antibodies), leading to the spread of glomerular inflammation [30]. Besides, miR-145 has been proved to affect VSMC differentiation via posttranscriptional suppression of proliferation-related proteins and thus has been involved in vascular diseases [31, 32]. Furthermore, downregulated miR-145 was observed in the vascular walls accompanied with neointimal lesion formation and in cultured dedifferentiated VSMCs [33]. In line with our result, Ding et al. [25] also confirmed that the miR-145 expression decreased with the increase in tunicae media vasorum ratio. Restoring miR-145 in lipopolysaccharides-stimulated cells by addition of a miR-145 mimic protected MH7A cells against lipopolysaccharides-induced apoptosis and inflammation [34].

In addition, with the further treatment of PDGF-BB, we also made conclusion that PDGF-BB treatment can suppress miR-145 expression in HVSMC. In addition, proliferation, migration and differentiation phenotype transformation (presented with increased $\alpha$-SMactin and decreased OPN) of HVSMCs induced by PDGF-BB were inhibited by transfection of miR-145, and overexpression of PDGF-BB from glomerular mesangial cells is the upstream mechanism for RVL in juvenile lupus nephritis. PDGF-BB is a well-known regulator of mesangial cells, which is involved in nephropathy and related not only to proinflammatory but also anti-inflammatory effects [35]. Previous evidence showed that patients with IgAN had significantly increased serum PDGF-DD levels, particularly in those with early disease [36]. Additionally, VSMC proliferation and migration can be enhanced by various growth factors and cytokines, including PDGF-BB [37]. Interestingly, decreased miR-145 is found in dedifferentiated VSMCs and is also a critical regulator of proliferation and migration of VSMCs, while overexpressed miR-145 can promote cell differentiation and inhibit VSMCs proliferation [31, 32]. Previous data have indicated the ability of PDGF-BB with regard to inhibition of miR-145-5p in VSMCs, and miR-145-5p can suppress the proliferation and migration of VSMCs induced by PDGF [38]. The phenotype change of VSMC is accompanied by the accelerated migration and accumulation of extracellular matrix, resulting in the injury of neointima, which is one of the causes of various proliferative vascular lesions [39]. Verification has been previously presented indicating that miR-145 plays an important role in the expression of the VSMC contractile phenotype due to its ability of regulating the stretchinduced differentiation of VSMCs [40, 41]. Moreover, miR-145 can regulate the phenotype switch of the VSMCs in the renal interstitium [25]. PDGF was also reported to promote the proliferation of VSMCs and increase OPN expression, which inhibit $\alpha$-SMA expression; 
however, these effects of PDGF were reversed by overexpressed miR-145 [42], which was consistent with our study.

With the involvement of 49 pediatric patients with $\mathrm{LN}$, we detected the expression of miR-145 in intrarenal vessels, and the regulatory role of miR-145 in HVSMCs was also investigated. All in all, our study demonstrated that the expression of miR-145 decreased with the increase in vascular damage. miR-145 can inhibit proliferation, migration, and differentiation phenotypic transformation of HVSMCs induced by PDGF-BB (Fig. 9). miR-145 may be involved in the pathogenesis of RVLs and may be a new target for treatment of RVLs in LN.

\section{Disclosure Statement}

The authors declare that they have no conflicts of interest.

\section{Funding Sources}

This work was supported by Hainan Natural Science Foundation Innovation Research Team Project (2018CXTD350), National Natural Science Foundation of China (Nos. 81260139, 81060073, 81560275, 61562021, 30560161), Hainan Special Projects of Social Development (2015SF39), Hainan Association for academic excellence Youth Science and Technology Innovation Program (201515), Hainan Natural Science Foundation of China (805106), and Shenzhen Science and Technology Research Fund (JCYJ20140414110526098).

\section{References}

1 Yang C, Xue J, An N, Huang XJ, Wu ZH, Ye L, et al. Accelerated Glomerular Cell Senescence in Experimental Lupus Nephritis. Med Sci Monit. 2018 Sep;24:6882-91.

2 Zununi Vahed S, Nakhjavani M, Etemadi J, Jamshidi H, Jadidian N, Pourlak T, et al. Altered levels of immuneregulatory microRNAs in plasma samples of patients with lupus nephritis. Bioimpacts. 2018;8(3):177-83.

3 Hanly JG, O'Keeffe AG, Su L, Urowitz MB, Romero-Diaz J, Gordon C, et al. The frequency and outcome of lupus nephritis: results from an international inception cohort study. Rheumatology (Oxford). 2016 Feb;55(2):252-62.

4 Saxena R, Mahajan T, Mohan C. Lupus nephritis: current update. Arthritis Res Ther. 2011;13(5):240.

5 Li Y, Fang X, Li QZ. Biomarker profiling for lupus nephritis. Genomics Proteomics Bioinformatics. 2013 Jun; 11(3):158-65.

6 Gargiulo ML, Khoury M, Gómez G, Grimaudo S, Suárez L, Collado MV, et al. Cut-off values of immunological tests to identify patients at high risk of severe lupus nephritis. Medicina (B Aires). 2018;78(5):329-35.

7 Liu CC, Manzi S, Ahearn JM. Biomarkers for systemic lupus erythematosus: a review and perspective. Curr Opin Rheumatol. 2005 Sep;17(5):543-9.

8 Liu X, Zhang Y, Wang S, Liu G, Ruan L. Loss of miR-143 and miR-145 in condyloma acuminatum promotes cellular proliferation and inhibits apoptosis by targeting NRAS. R Soc Open Sci. 2018 Aug;5(8):172376.

9 Ishii H, Vodnala SK, Achyut BR, So JY, Hollander MC, Greten TF, et al. miR-130a and miR-145 reprogram Gr-1+CD11b+ myeloid cells and inhibit tumor metastasis through improved host immunity. Nat Commun. 2018 Jul;9(1):2611.

10 Mukhadi S, Hull R, Mbita Z, Dlamini Z. The Role of MicroRNAs in Kidney Disease. Noncoding RNA. 2015 Nov; 1(3):192-221.

11 Han X, Wang Y, Zhang X, Qin Y, Qu B, Wu L, et al. MicroRNA-130b Ameliorates Murine Lupus Nephritis Through Targeting the Type I Interferon Pathway on Renal Mesangial Cells. Arthritis Rheumatol. 2016 Sep;68(9): 2232-43.

12 Zhao X, Zhang W, Ji W. MY05A inhibition by miR-145 acts as a predictive marker of occult neck lymph node metastasis in human laryngeal squamous cell carcinoma. OncoTargets Ther. 2018 Jun;11:3619-35.

13 Cordes KR, Sheehy NT, White MP, Berry EC, Morton SU, Muth AN, et al. miR-145 and miR-143 regulate smooth muscle cell fate and plasticity. Nature. 2009 Aug;460(7256):705-10.

14 Xin M, Small EM, Sutherland LB, Qi X, McAnally J, Plato CF, et al. MicroRNAs miR-143 and miR-145 modulate cytoskeletal dynamics and responsiveness of smooth muscle cells to injury. Genes Dev. 2009 Sep;23(18): 2166-78. 


\begin{tabular}{l|l}
\hline Kidney Blood Press Res 2019;44:643-655 \\
\hline DOI: 10.1159/000500923 & $\begin{array}{l}\text { (c) 2019 The Author(s). Published by S. Karger AG, Basel } \\
\text { www.karger.com/kbr }\end{array}$ \\
\hline
\end{tabular}

Cai et al.: miR-145 in RVLs in Patients with JLN

15 Boettger T, Beetz N, Kostin S, Schneider J, Krüger M, Hein L, et al. Acquisition of the contractile phenotype by murine arterial smooth muscle cells depends on the Mir143/145 gene cluster. J Clin Invest. 2009 Sep;119(9): 2634-47.

16 Zhu X, Zhu R. Curcumin suppresses the progression of laryngeal squamous cell carcinoma through the upregulation of miR-145 and inhibition of the PI3K/Akt/mTOR pathway. OncoTargets Ther. 2018 Jun;11:3521-31.

17 Ding Y, Zhang C, Zhang J, Zhang N, Li T, Fang J, et al. miR-145 inhibits proliferation and migration of breast cancer cells by directly or indirectly regulating TGF- $\beta 1$ expression. Int J Oncol. 2017 May;50(5):1701-10.

18 Wang L, Wu X, Wang B, Wang Q, Han L. Mechanisms of miR-145 regulating invasion and metastasis of ovarian carcinoma. Am J Transl Res. 2017 Jul;9(7):3443-51.

19 Wang W, Ji G, Xiao X, Chen X, Qin WW, Yang F, et al. Epigenetically regulated miR-145 suppresses colon cancer invasion and metastasis by targeting LASP1. Oncotarget. 2016 0ct;7(42):68674-87.

20 Collison A, Mattes J, Plank M, Foster PS. Inhibition of house dust mite-induced allergic airways disease by antagonism of microRNA-145 is comparable to glucocorticoid treatment. J Allergy Clin Immunol. 2011 Jul; 128(1):160-7.e4.

21 Appel GB, Pirani CL, D’Agati V. Renal vascular complications of systemic lupus erythematosus. J Am Soc Nephrol. 1994 Feb;4(8):1499-515.

22 Tangtanatakul P, Klinchanhom S, Sodsai P, Sutichet T, Promjeen C, Avihingsanon Y, et al. Down-regulation of let-7a and miR-21 in urine exosomes from lupus nephritis patients during disease flare. Asian Pac J Allergy Immunol. DOI: 10.12932/AP-130318-0280.

23 Zhao P, Miao J, Zhang K, Lv M, Han Q, Zhu P. Circulating Angiogenic T Cells Are Increased in Lupus Nephritis Patients. Med Sci Monit. 2018 Aug;24:5384-90.

24 Pauley KM, Cha S, Chan EK. MicroRNA in autoimmunity and autoimmune diseases. J Autoimmun. 2009 May-Jun;32(3-4):189-94.

25 Ding Y, Liao W, Yi Z, Xiang W, He X. Association of miRNA-145 expression in vascular smooth muscle cells with vascular damages in patients with lupus nephritis. Int J Clin Exp Pathol. 2015 0ct;8(10):12646-56.

26 Weening JJ, D’Agati VD, Schwartz MM, Seshan SV, Alpers CE, Appel GB, et al. The classification of glomerulonephritis in systemic lupus erythematosus revisited. J Am Soc Nephrol. 2004 Feb;15(2):241-50.

27 Avihingsanon Y, Benjachat T, Tassanarong A, Sodsai P, Kittikovit V, Hirankarn N. Decreased renal expression of vascular endothelial growth factor in lupus nephritis is associated with worse prognosis. Kidney Int. 2009 Jun;75(12):1340-8.

28 Froy 0, Sthoeger ZM. Defensins in systemic lupus erythematosus. Ann N Y Acad Sci. 2009 Sep;1173(1):365-9.

29 Chen JY, Wang CM, Chen TD, Jan Wu YJ, Lin JC, Lu LY, et al. Interferon- $\lambda 3 / 4$ genetic variants and interferon- $\lambda 3$ serum levels are biomarkers of lupus nephritis and disease activity in Taiwanese. Arthritis Res Ther. 2018 Aug;20(1):193.

30 Gatto M, Iaccarino L, Ghirardello A, Punzi L, Doria A. Clinical and pathologic considerations of the qualitative and quantitative aspects of lupus nephritogenic autoantibodies: a comprehensive review. J Autoimmun. 2016 May;69:1-11.

31 Wu J, Song HF, Li SH, Guo J, Tsang K, Tumiati L, et al. Progressive Aortic Dilation Is Regulated by miR-17-Associated miRNAs. J Am Coll Cardiol. 2016 Jun;67(25):2965-77.

32 Cheng Y, Liu X, Yang J, Lin Y, Xu DZ, Lu Q, et al. MicroRNA-145, a novel smooth muscle cell phenotypic marker and modulator, controls vascular neointimal lesion formation. Circ Res. 2009 Jul;105(2):158-66.

33 Girdauskas E, Petersen J, Neumann N, Ungelenk M, Kurth I, Reichenspurner H, et al. MiR-145 expression and rare NOTCH1 variants in bicuspid aortic valve-associated aortopathy. PLoS One. 2018 Jul;13(7):e0200205.

34 Zhong F, Xu J, Yang X, Zhang Q, Gao Z, Deng Y, et al. miR-145 eliminates lipopolysaccharides-induced inflammatory injury in human fibroblast-like synoviocyte MH7A cells. J Cell Biochem. 2018 Dec;119(12):10059-66.

35 Mei HE, Hahne S, Redlin A, Hoyer BF, Wu K, Baganz L, et al. Plasmablasts with a Mucosal Phenotype Contribute to Plasmacytosis in Systemic Lupus Erythematosus. Arthritis Rheumatol. 2017 Oct;69(10):2018-28.

36 Boor P, Eitner F, Cohen CD, Lindenmeyer MT, Mertens PR, Ostendorf T, et al.; ERCB-Consortium. Patients with IgA nephropathy exhibit high systemic PDGF-DD levels. Nephrol Dial Transplant. 2009 Sep;24(9):2755-62.

37 Yi N, Chen SY, Ma A, Chen PS, Yao B, Liang TM, et al. Tunicamycin inhibits PDGF-BB-induced proliferation and migration of vascular smooth muscle cells through induction of HO-1. Anat Rec (Hoboken). 2012 Sep;295(9): 1462-72.

38 Li L, Mao D, Li C, Li M. miR-145-5p Inhibits Vascular Smooth Muscle Cells (VSMCs) Proliferation and Migration by Dysregulating the Transforming Growth Factor-b Signaling Cascade. Med Sci Monit. 2018 Jul;24:4894-904.

39 Sozeri B, Deveci M, Dincel N, Mir S. The early cardiovascular changes in pediatric patients with systemic lupus erythematosus. Pediatr Nephrol. 2013 Mar;28(3):471-6.

40 Hu B, Song JT, Qu HY, Bi CL, Huang XZ, Liu XX, et al. Mechanical stretch suppresses microRNA-145 expression by activating extracellular signal-regulated kinase $1 / 2$ and upregulating angiotensin-converting enzyme to alter vascular smooth muscle cell phenotype. PLoS One. 2014 May;9(5):e96338.

41 Turczynska KM, Sadegh MK, Hellstrand P, Swärd K, Albinsson S. MicroRNAs are essential for stretch-induced vascular smooth muscle contractile differentiation via microRNA (miR)-145-dependent expression of L-type calcium channels. J Biol Chem. 2012 Jun;287(23):19199-206.

42 Li Y, Huang J, Jiang Z, Zhong Y, Xia M, Wang H, et al. MicroRNA-145 regulates platelet-derived growth factorinduced human aortic vascular smooth muscle cell proliferation and migration by targeting CD 40. Am J Transl Res. 2016 Apr;8(4):1813-25. 